\title{
Afecções Pericárdicas em Pacientes com COVID-19: Uma Possível Causa de Deterioração Hemodinâmica
}

\author{
Pericardial Affections in Patients with COVID-19: A Possible Cause of Hemodynamic Deterioration
}

\author{
Fábio Fernandes, ${ }^{1 \oplus}$ Felix José Alvarez Ramires, ${ }^{1 \oplus}$ Fábio Danziato Fernandes, ${ }^{2}$ Marcus Vinicius Simões, ${ }^{3 \oplus}$ Evandro \\ Tinoco Mesquita, ${ }^{4}$ Charles Mady ${ }^{1}$ \\ Universidade de São Paulo Faculdade de Medicina Hospital das Clínicas Instituto do Coração, ${ }^{1}$ São Paulo, SP - Brasil \\ Centro Universitário São Camilo, ${ }^{2}$ São Paulo, SP - Brasil \\ Universidade de São Paulo Faculdade de Medicina de Ribeirão Preto, ${ }^{3}$ Ribeirão Preto, SP - Brasil \\ Universidade Federal Fluminense, ${ }^{4}$ Niterói, RJ - Brasil
}

\section{Introdução}

O curso clínico da infecção por SARS-CoV-2 é caracterizado por sintomas do trato respiratório, febre, tosse, dor de garganta, fadiga e complicações relacionadas à síndrome do desconforto respiratório agudo. No entanto, alguns pacientes que inicialmente apresentam sintomas leves poderão subsequentemente apresentar deterioração clínica, a qual ocorre aproximadamente uma semana após o início dos sintomas. ${ }^{1}$

A progressão aguda da COVID-19 pode ser dividida em três fases distintas: infecção precoce, fase pulmonar e hiperinflamação. No entanto, pode haver sobreposição significativa entre as diferentes fases em qualquer paciente. ${ }^{2}$

O envolvimento cardíaco é uma característica proeminente da COVID-19 e está associado a pior prognóstico. Os mecanismos de lesão cardíaca são múltiplos, sendo descritos aumento do estresse cardíaco secundário a insuficiência respiratória, hipoxemia, infecção miocárdica direta pelo SARSCoV-2, doença microvascular ou microtrombose e injúria indireta secundária a inflamação sistêmica. ${ }^{3}$

Frente a um paciente com COVID-19 que apresenta piora clínica, devemos incluir como possíveis diagnósticos diferenciais: pneumonia em evolução pelo SARS-CoV-2, exacerbação aguda da insuficiência cardíaca crônica, síndrome coronariana aguda, embolia pulmonar aguda, miocardite e afecções pericárdicas. ${ }^{4}$

Diante do exposto, realizou-se uma revisão não sistemática da literatura a fim de abordar os principais estudos que sugerem comprometimento pericárdico em pacientes com

\section{Palavras-chave}

Coronavirus-19; COVID-19; Betacoronavirus/ complicações; Pandemia; SARS-CoV-2; Hipóxia; Complicações Cardiovasculares; Inflamação Sistêmica; Trombose.

\section{Correspondência: Fábio Fernandes •}

Universidade de São Paulo Faculdade de Medicina Hospital das Clínicas Instituto do Coração - Av. Dr. Eneas C. Aguiar, 44. CEP 05403-000, São Paulo, SP - Brasil

E-mail: fabio.fernandes@incor.usp.br

Artigo recebido em 14/05/2020, revisado em 17/07/2020,

aceito em 29/07/2020

DOI: https://doi.org/10.36660/abc.20200474
COVID-19. A base de dados consultada foi a PubMed (www. ncbi.nlm.nih.gov/pubmed). Foram selecionados artigos originais e de revisão realizados em humanos, redigidos em português e inglês. Os seguintes descritores MeSH foram utilizados como palavras-chave: COVID-19, cardiac tamponade, SARS-CoV-2 e pericarditis.

Neste trabalho, objetivamos revisar as evidências disponíveis até o momento em relação ao envolvimento pericárdico de pacientes com SARS-CoV-2, suas formas de apresentação clínica, mecanismos fisiopatológicos e possibilidades terapêuticas, salientando a possiblidade de comprometimento hemodinâmico pela ocorrência de tamponamento cardíaco (Quadro 1).

\section{Afecções Pericárdicas}

O derrame pericárdico é a apresentação clínica mais comum das pericardiopatias. Processos infecciosos causados por agentes virais, bacterianos ou fúngicos podem cursar ou não com o acometimento do pericárdio. Em caso de pericardite, encontraremos, em sua forma aguda, manifestações clínicas como dor torácica tipo pleurítica e atrito pericárdico, além de alterações no traçado

\section{Quadro 1 - Reconhecimento de pericardiopatias em pacientes com} COVID-19

1. Paciente com COVID-19 e suspeita de pericardite:

- dispneia, taquipneia, edema de membros inferiores;

- taquicardia;

- hipotensão;

- pulso paradoxal.

2. Exames complementares:

- ECG (baixa voltagem);

- troponina e CKMB (miopericardite);

- ecocardiograma transtorácico (sinais de tamponamento).

3. Diagnóstico diferencial:

- síndrome coronariana aguda;

- cardiomiopatia relacionada a sepse (tempestade de citocinas);

- miocardite;

- síndrome de Takotsubo.

4. Investigações adicionais:

- ressonância magnética cardíaca, se possível;

- angiotomografia de coronárias.

5. Considerar periocardiocentese 


\section{Carta Científica}

eletrocardiográfico. A condição mais comumente associada ao derrame pericárdico é a pericardite aguda de etiologia viral, frequentemente uma condição autolimitada. ${ }^{5}$

Nos casos de pacientes com COVID-19, a ocorrência de derrame pleural, linfadenopatia, cavitação, sinal de halo da tomografia de tórax, pneumotórax e derrame pericárdico é incomum, mas pode ser observada com a progressão da doença. ${ }^{6} \mathrm{~A}$ ação do vírus no pericárdio pode ser por efeito citotóxico direto e/ou por mecanismo imunomediado. ${ }^{7}$

O primeiro relato de tamponamento cardíaco secundário à infecção pela COVID-19 foi feito em 30 março de 2020, em uma paciente de 47 anos (Quadro 2). A paciente referia sintomas de febre e tosse seca e não apresentava comorbidades cardiovasculares, como hipertensão ou diabetes, mas apresentava antecedente de miopericardite e teste positivo para COVID-19 em swab nasofaríngeo. Evoluiu com instabilidade hemodinâmica, sendo diagnosticado tamponamento cardíaco e identificada necessidade de pericardiocentese - na qual $540 \mathrm{~mL}$ de líquido serossanguinolento foram retirados. Esse caso chamou atenção da comunidade médica para a possibilidade do comprometimento pericárdico evoluir para tamponamento cardíaco e ser um fator responsável pela deterioração hemodinâmica em pacientes com COVID-19. ${ }^{8}$

Outro caso de tamponamento pericárdico foi identificado em uma paciente de 67 anos com antecedente de miocardiopatia isquêmica e fração de ejeção de $40 \%$ que, após uma semana do início de sintomas respiratórios, desenvolveu fadiga, hipotensão e taquicardia. Além disso, o eletrocardiograma evidenciou baixa voltagem, com os marcadores séricos troponina e BNP normais. Devido à persistência dos sintomas, realizou-se ecocardiograma, o qual evidenciou tamponamento pericárdico. Em seguida, foi realizada drenagem de $800 \mathrm{~mL}$ de líquido serohemorrágico com características de exsudato. Logo após a pericardiocentese, a paciente evoluiu para cardiomiopatia induzida por estresse, caraterizada pelo abaulamento apical transitório (síndrome de Takotsubo), conforme achados clínicos, laboratoriais, eletro e ecocardiográficos. ${ }^{7}$
Uma paciente de 53 anos, sem antecedentes mórbidos, procurou serviço uma semana após o início dos sintomas. Deu entrada com hipotensão e alterações eletrocardiográficas com supradesnivelamento difuso do segmento ST e elevação de biomarcadores, como troponina e NT-proBNP, mas com coronárias angiograficamente normais. Foi solicitado exame PCR de swab de nasofaringe, o qual testou positivo para SARS-CoV-2. A ressonância magnética cardíaca evidenciou edema e realce tardio miocárdicos, além de derrame pericárdico circunferencial compatível com miopericardite. ${ }^{9}$

Um paciente de 59 anos deu entrada com síndrome coronariana aguda e foi submetido a cateterismo cardíaco, o qual evidenciou lesões triarteriais. O paciente recebeu tratamento de revascularização miocárdica. No 22ํㅜ pósoperatório, iniciou sintomas de febre e dispneia e apresentou PCR positivo para SARS-CoV-2, com tomografia de tórax compatível com pneumonia por SARS-CoV-2. ${ }^{10}$ No 23 o dia de infecção, o paciente evoluiu com dor precordial, dispneia, hipotensão e taquicardia. O ecocardiograma evidenciou derrame pericárdico circunferencial com sinais de tamponamento de câmaras direitas, sendo drenados 250 $\mathrm{mL}$ de líquido serossanguinolento.

Em outro caso, uma paciente de 70 anos com antecedentes de doença arterial coronária aguda (infarto do miocárdio sem supradesnivelamento do segmento ST duas semanas antes da internação, com angioplastia recente) deu entrada com sintomas respiratórios. No segundo dia de internação, apresentou dor torácica sugestiva de pericardite aguda, evoluindo com tamponamento cardíaco, intubação orotraqueal e instabilidade hemodinâmica com necessidade de drogas vasoativas. Houve melhora clínica e hemodinâmica apenas após a pericardiocentese..$^{11}$

Outro caso de COVID-19 e tamponamento cardíaco envolveu uma paciente de 41 anos, sem antecedentes prévios, que havia procurado o serviço com sintomas respiratórios 10 dias antes da internação. Realizou-se pericardiocentese e evidenciou-se exsudato no líquido pericárdico, com elevados níveis de DHL e albumina. ${ }^{12}$

Quadro 2 - Casos de afecções pericárdicas e COVID-19

\begin{tabular}{|c|c|c|c|c|c|c|}
\hline $\begin{array}{l}\text { Data publicação (ref.) } \\
\text { País }\end{array}$ & $\begin{array}{l}\text { Idade } \\
\text { (anos) }\end{array}$ & Sexo & Antecedentes CV & $\begin{array}{l}\text { Tamponamento/ } \\
\text { Pericardiocentese }\end{array}$ & $\begin{array}{l}\text { Sintomas } \\
\text { prévios } \\
\text { COVID-19 }\end{array}$ & Tratamento coadjuvante \\
\hline $\begin{array}{l}\text { 30/03/2020 } \\
\text { Reino Unido }\end{array}$ & 47 & $\mathrm{~F}$ & Miopericardite & $\mathrm{Sim} / \mathrm{Sim}$ & Sim & Não relatado \\
\hline $\begin{array}{l}27 / 03 / 2020 \\
\text { Itália }^{9}\end{array}$ & 53 & $\mathrm{~F}$ & Sem antecedentes & $\begin{array}{c}\text { Não / Não } \\
\text { (miopericardite) }\end{array}$ & Sim & $\begin{array}{l}\text { Lopinavir/Ritonavir } \\
\text { Corticoide/hidroxicloroquina }\end{array}$ \\
\hline $\begin{array}{l}\text { 23/04/2020 } \\
\text { Itália }^{10}\end{array}$ & 59 & M & $\begin{array}{l}\text { Cardiopatia isquêmica aguda } \\
\text { (revascularização miocárdica) }\end{array}$ & $\operatorname{Sim} / \operatorname{Sim}$ & Sim & $\begin{array}{c}\text { Lopinavir/Ritonavir } \\
\text { Enoxaparina/hidroxicloroquina }\end{array}$ \\
\hline $\begin{array}{l}\text { 23/04/2020 } \\
\text { Estados Unidos }\end{array}$ & 67 & $\mathrm{~F}$ & $\begin{array}{l}\text { Miocardiopatia isquêmica } \\
\text { FE } 40 \%\end{array}$ & Sim / Sim & Sim & Colchicina, hidroxicloroquina, corticoide \\
\hline $\begin{array}{l}\text { 12/05/202011 } \\
\text { Arábia Saudita }\end{array}$ & 41 & $\mathrm{~F}$ & Sem antecedentes & $\operatorname{Sim} / \operatorname{Sim}$ & Sim & Azitromicina e Hidroxicloroquina \\
\hline $\begin{array}{l}\text { 06/05/2020 } \\
\text { Estados Unidos }\end{array}$ & 70 & $\mathrm{~F}$ & $\begin{array}{l}\text { Cardiopatia isquêmica, } \\
\text { hipertensão e diabetes }\end{array}$ & $\operatorname{Sim} / \operatorname{Sim}$ & Sim & Colchicina \\
\hline
\end{tabular}


Nesses seis primeiros casos, observamos que pacientes com SARS-CoV-2 podem apresentar comprometimento pericárdico na evolução clínica. O período de diagnóstico e piora clínica, em geral após a primeira semana da infecção, coincide com a fase de aumento de citocinas inflamatórias, provavelmente com mecanismos autoimunes envolvidos no mecanismo etiopatogênico do derrame.

Alguns autores acreditam que, em pacientes com miocardite secundária ao coronavírus, a infecção viral pode ser o fator inicial, desencadeando injúria imunomediada. Em estudos de necropsia, observa-se infiltrado inflamatório mononuclear nos tecidos cardíacos, porém sem presença de inclusão viral em alguns casos. ${ }^{13}$ A rápida recuperação da função cardíaca e o leve aumento na carga viral sugerem que, além da replicação viral no miocárdio, é possível que a resposta imune, uma tempestade de citocinas, realmente possa desempenhar um papel significativo na fisiopatologia da agressão. ${ }^{14}$

A liberação excessiva de citocinas pode ser observada em grande variedade de doenças sistêmicas, incluindo doenças infecciosas, reumáticas e neoplásicas, as quais podem levar ao comprometimento pericárdico. ${ }^{15}$

Imunologistas e patologistas observaram infiltrado de macrófagos na necropsia, podendo indicar que a viremia e imunidade inata dominam o quadro clínico antes que o infiltrado linfocitário possa ocorrer. Observam-se parâmetros inflamatórios aumentados, entre eles a proteína C-reativa e as citocinas pró-inflamatórias (IL-6, TNF $\alpha$, IL-8I). ${ }^{15}$

Dessa forma, um estado hiperinflamatório induzido pela ação de citocinas pode levar à insuficiência de múltiplos órgãos e poderia ser responsável pelo comprometimento miocárdico e pericárdico na doença; no entanto, não podemos excluir a possibilidade de parte desse comprometimento ser causado pelo vírus. ${ }^{16}$

O caso do paciente relatado no estudo italiano exibiu um período mais tardio até o aparecimento de pericardite, e a análise química e citológica do líquido pericárdico demonstrou presença de infiltrado inflamatório com predomínio linfocitário e presença de SARS-CoV-2, sugerindo possível efeito direto do vírus nas manifestações cardiológicas. É interessante notar que o paciente apresentava PCR negativo no sangue e na região nasofaríngea, sendo apenas positivo no líquido pericárdico, o que pode sugerir possível reservatório do vírus no pericárdio. ${ }^{10}$

Em cinco dos casos, a apresentação foi de tamponamento cardíaco, uma causa de descompensação clínica de alto risco, mas potencialmente reversível. A decisão de adotar uma técnica invasiva de tratamento é influenciada pelo quadro clínico do paciente. Quando o quadro for evidente, com presença de hipotensão arterial, dispneia e pulso paradoxal, não existe controvérsia quanto ao procedimento. A conduta frente ao derrame pericárdico deve estar embasada no contexto clínico (piora dos sintomas), hemodinâmico (presença ou não de tamponamento) ou pesquisa da etiologia (infecção, tuberculose ou neoplasia). ${ }^{5}$

\section{Tratamento Farmacológico}

A definição do melhor tratamento farmacológico para afecção pericárdica em pacientes com SARS-CoV-2 merece discussão. Os anti-inflamatórios não hormonais (AINHs) estão indicados como primeira opção em todos os casos de pericardite aguda e recorrente, desde que não apresentem contraindicações. Recomenda-se ácido acetilsalicílico (AAS) 800 mg, a cada oito horas, ou ibuprofeno 600 mg, a cada oito horas, em associação com a colchicina. ${ }^{5}$

Um estudo retrospectivo realizado na França ${ }^{17}$ demonstrou que o uso de AINHs para efeito de sintomas prévio à hospitalização por pneumonia desenvolveu formas mais graves da doença e período maior de internação dos pacientes. Por outro lado, o uso de ibuprofeno e acetaminofeno também esteve associado a maior risco de complicações em crianças, principalmente em altas doses cumulativas. Especula-se que o alívio sintomático da dor e febre possa acarretar retardo na introdução precoce de antibióticos. ${ }^{18}$ Os possíveis vieses de que pacientes com infecções virais mais severas, como influenza e COVID-19, são mais propensos a utilizar anti-inflamatórios e ibuporofeno poderiam ser fatores confundidores do risco aumentado.

A controvérsia relacionada à segurança do uso de ibuprofeno em pacientes com COVID-19 originou-se na França, quando um infectologista relatou piora dos sintomas em quatro pacientes que haviam feito uso da medicação. O relato foi logo respaldado pelo Ministério da Saúde Francês. ${ }^{19,20}$ Por outro lado, há dados que mostram que o ibuprofeno aumenta a expressão de receptores $\mathrm{ACE}_{2}$ No entanto, sabemos que evidências de efeitos mecanísticos nem sempre são confirmados por estudos clínicos. ${ }^{20}$ Atualmente, as recomendações da Organização Mundial da Saúde e da Food and Drug Administration (FDA) não se opõem ao uso de ibuprofeno em casos sintomáticos de COVID-19. ${ }^{21} \mathrm{Em}$ resumo, as evidências epidemiológicas recentes não são suficientes para afirmar um elo causal entre efeito deletério e uso de ibuprofeno em pacientes com COVID-19. Dessa forma, deve-se ponderar a relação risco-benefício na decisão sobre o uso desse medicamento em pacientes com COVID-19. ${ }^{22}$

Nos últimos anos, a colchicina vem se destacando como medicação coadjuvante aos AINH tanto no tratamento da pericardite aguda quanto no da recorrente, com excelentes resultados, devendo também ser considerada no tratamento da pericardite em pacientes com SARS-CoV-2. Os efeitos anti-inflamatórios da colchicina estão relacionados à ruptura da função dos microtúbulos, cujo resultado é a inibição dos neutrófilos e moléculas de adesão que interferem no início e na amplificação da inflamação. ${ }^{5}$ Com relação ao uso da colchicina em pacientes com COVID-19, existem pelo menos oito estudos incluídos no site https://clinicaltrials.gov/ com o objetivo de testar o efeito do medicamento em atenuar a inflamação sistêmica e/ou miocárdica. Dois dos estudos já estão em andamento: Colchicine Coronavirus SARS-CoV2 Trial (COLCORONA) e The ECLA PHRI COLCOVID Trial. Effects of Colchicine on Moderate/High-risk Hospitalized COVID-19 Patients.

Os corticosteroides são muito utilizados no tratamento da pericardite aguda e de suas recorrências porque melhoram os sintomas e causam diminuição de marcadores inflamatórios. Porém, seu uso é limitado aos casos de intolerância, contraindicações ou falha do tratamento com AINHs e colchicina devido ao aumento de recorrências, como 
demonstrado no estudo COPE. ${ }^{5}$ Há também indicação para o uso de corticosteroides em casos de etiologias específicas, como doenças autoimunes, porém nas menores doses possíveis. Nessas situações, a diminuição da dose deve ser bem lenta e iniciar apenas após o desaparecimento dos sintomas e a normalização da proteína C-reativa. Doses de 0,25 a $0,5 \mathrm{mg} / \mathrm{kg} /$ dia devem ser utilizadas nos casos de pericardite. ${ }^{5}$

De maneira geral, para as afecções pericárdicas, o uso de corticoides em detrimento do uso de AINHs e colchicina é sempre evitado. Contudo, a COVID-19 tem se mostrado uma doença peculiar com características fisiopatológicas distintas, na qual há um estado de hiperinflamação e tempestade de citocinas. Nesses casos, a introdução de corticoides talvez possa ser considerada como primeira opção no tratamento das afecções pericárdicas em pacientes com COVID-19. Para essa doença, fármacos que reduzem a inflamação antes da disfunção de múltiplos-sistemas têm sido preconizados - dessa forma, o uso de corticoides poderia ser justificado. Porém, permanecem dúvidas referentes à dose e ao tempo de tratamento ideais. ${ }^{16}$

Diretrizes internacionais sobre o manejo e tratamento da sepse e do choque séptico recomendam que corticoides sejam utilizados em doses pequenas, por curto período de tempo, e nos casos em que a reposição volêmica e as drogas vasopressoras não estabilizarem o paciente. ${ }^{23}$ Em um estudo de Chen et al., ${ }^{24}$ 19\% dos pacientes com pneumonia receberam corticoides - metilprednisolona, 1-2 $\mathrm{mg} / \mathrm{kg}$ por dia, e dexametasona, por 3 a 15 dias (mediana de 5 dias). Os potenciais riscos do tratamento com corticoides seriam o retardo na eliminação viral e a ocorrência de infecções secundárias. ${ }^{15}$

O SARS-CoV-2 foi inicialmente identificado no lavado pulmonar coletado de um paciente com pneumonia, no final de dezembro de 2019. Desde então, tem-se isolado o vírus no trato respiratório, nas fezes, na conjuntiva e no sangue. Recentemente, tivemos relato da presença do vírus no líquido pericárdico de um paciente com tamponamento cardíaco submetido a pericardiocentese..$^{10}$ Dessa forma, o uso de corticoides seria especulativo nessa situação.

Observamos que alguns pacientes com afecção pericárdica e COVID-19 utilizaram hidroxicloroquina como parte do tratamento da pericardite. No entanto, não há evidência da eficácia e segurança desse medicamento nos pacientes. Pacientes com COVID-19 apresentam quadro de inflamação sistêmica e muitos desenvolvem miocardite concomitante; tal quadro inflamatório pode levar a arritmias ventriculares graves, e a introdução de hidroxicloroquina, um medicamento que aumenta o intervalo QT, poderia potencializar a ocorrência de torsade de points.

O tocilizumab, um anticorpo monoclonal antirreceptor de IL-6, está sendo testado em estudos multicêntricos randomizados em pacientes com COVID-19 que apresentam aumento de IL-6 e que possam se beneficiar do tratamento em casos de síndrome de tempestade de citocinas, reduzindo a inflamação miocárdica.
Outra característica clínica proeminente em pacientes com as formas graves de COVID-19 são as manifestações de lesão endotelial e trombose microvascular, podendo mimetizar vasculite, inclusive com casos de amputação de extremidades. No estudo patológico, observa-se infiltração de monócitos e linfócitos ao redor dos vasos sanguíneos com hiperplasia e espessamento da parede do vaso, além de trombos em microvasos; altos níveis de anticorpos antifosfolípides, anticardiolipina e anti- $\beta_{2}$ glicoproteína estiveram associados a trombose..$^{15}$ Dessa forma, alguns estudos recomendam o uso de anticoagulantes ${ }^{25}$ para tentar evitar fenômenos tromboembólicos e, como observado nos casos relatados anteriormente, os clínicos devem estar atentos, nessa situação, para o risco aumentado de ocorrência de hemopericárdio e tamponamento.

Recentemente, foi publicado um registro internacional de pacientes com casos suspeitos e confirmados de COVID-19 em 69 países de seis continentes, totalizando 1.216 casos. Os pacientes foram submetidos a ecocardiograma, e observou-se que a maioria dos casos apresentava anormalidades não específicas de disfunção ventricular. Entretanto, observaram-se novo infarto do miocárdio, miocardite e cardiomiopatia de Takotsubo em uma minoria dos casos. Em 11 casos (1\%), evidenciou-se presença de tamponamento pericárdico, o que demonstra a importância do tema e a possibilidade da utilização de métodos complementares, como o ecocardiograma, para identificar e modificar diretamente a conduta clínica e, consequentemente, o tratamento desses pacientes. ${ }^{26}$

A cardiovigilância dos pacientes que desenvolveram comprometimento pericárdico é de fundamental importância, com o objetivo de avaliar recorrências e/ou evolução para formas constritivas a longo prazo.

\section{Contribuição dos Autores}

Concepção e desenho da pesquisa: Fernandes F, Mesquita ET; Obtenção de dados: Fernandes F, Fernandes FD, Simões MV, Mesquita ET; Análise e interpretação dos dados e Revisão crítica do manuscrito quanto ao conteúdo intelectual importante: Fernandes F, Ramires F, Fernandes FD, Simões MV, Mesquita ET, Mady C; Redação do manuscrito: Fernandes F, Ramires F, Fernandes FD, Simões MV, Mesquita ET.

\section{Potencial Conflito de Interesses}

Declaro não haver conflito de interesses pertinentes.

\section{Fontes de Financiamento}

O presente estudo não teve fontes de financiamento externas.

\section{Vinculação Acadêmica}

Não há vinculação deste estudo a programas de pósgraduação. 


\section{Referências}

1. Wei X, Fang Y, Hu H. Immune-mediated mechanism in coronavirus fulminant myocarditis. Eur Heart J. 2020 Apr 22;41(19):1855.

2. Akhmerov A, Marban E. COVID-19 and the heart. Circ Res. 2020 May 8;126(10):1443-55.

3. Madjid M, Safavi-Naeini P, Solomon SD, Vardeny O. Potential effects of coronaviruses on the cardiovascular system: a review. JAMA Cardiol. 2020 Mar 27;5(7):831-40.

4. Cizgici AY, Zencirkiran Agus H, Yildiz M. COVID-19 myopericarditis: it should be kept in mind in today's conditions. Am J Emerg Med. 2020;S07356757(20)30310-7.

5. Adler Y, Charron P, Imazio M, Badano L, Barón-Esquivias G, Bogaert J, et al. 2015 ESC Guidelines for the diagnosis and management of pericardial diseases: The Task Force for the Diagnosis and Management of Pericardial Diseases of the European Society of Cardiology ( ESC) Endorsed by: The European Association of Cardio-Thoracic Surgery (EACTS). Eur Heart J. 2015;36(42):2921-64

6. Salehi S, Abedi A, Balakrishnan S, Gholamrezanezhad A. Coronavirus disease 2019 (COVID-19): A systematic review of imaging findings in 919 patients. AJR Am J Roentgenol. 2020 Mar 14;215(11):87-93.:1-7.

7. Dabbagh MF, Aurora L, D'Souza P, Weinmann AJ, Bhargava P, Basir MB Cardiac tamponade secondary to COVID-19. JACC Case Rep. 2020 Apr $23 ; 7(9): 326-30$

8. Hua A, O'Gallagher K, Sado D, Byrne J. Life-threatening cardiac tamponade complicating myo-pericarditis in COVID-19. Eur Heart J. 2020 Jun 7;41(22):2130.

9. Inciardi RM, Lupi L, Zaccone G, Italia L, et al. Cardiac involvement in a patient with coronavirus disease 2019 (COVID-19). JAMA Cardiol. 2020 March $27 ; 5(7):[$ online ahead of print]

10. Farina A, Uccello G, SpreaficoM, BassanelliG, Savonitto S. SARS-CoV-2 detection in the pericardial fluid of a patient with cardiac tamponade. Eur J Intern Med. 2020;76:100-101. doi: 10.1016/j.ejim.2020.04.045. Epub 2020 Apr 23.

11. Allam HH, Kinsara AJ, Tuaima T, Alfakih S. Pericardial fluid in a COVID-19 patient: is it exudate or transudate? Eur J Case Rep Intern Med. 2020;7(6):001703.

12. Asif T, Kassab K, Iskander F, Alyousef T. Acute pericarditis and cardiac tamponade in a patient with COVID-19: a therapeutic challenge. Eur J Case Rep Intern Med. 2020;7(6):001701.

13. Xu Z, Shi L, Wang Y, Zhang J, Huang L, Zhang C, et al. Pathological findings of COVID-19 associated with acute respiratory distress syndrome. Lancet Respir Med. 2020 Apr;8(4):420-22.
14. Channappanavar R, Perlman S. Pathogenic human coronavirus infections: causes and consequences of cytokine storm and immunopathology. Semin Immunopathol. 2017;39(5):529-39.

15. Zhang W, Zhao Y, Zhang F, Wang Q, Li T, Liu Z, et al. The use of antiinflammatory drugs in the treatment of people with severe coronavirus disease 2019 (COVID-19): the perspectives of clinical immunologists from China. Clin Immunol. 2020 May; 214:108393.

16. Maisch B, Dörr R. COVID-19-What we know and what we need to know: there are more questions than answers. Herz. 2020 Jun;45(4):311-2.

17. Kotsiou OS, Zarogiannis SG, Gourgoulianis KI. Prehospital NSAIDs use prolong hospitalization in patients with pleuro-pulmonary infection. Respir Med. 2017;123:28-33.

18. Krenke K, Krawiec M, Kraj G, Peradzynska J, Krauze A, Kulus M. Risk factors for local complications in children with community-acquired pneumonia. Clin Respir J. 2018;12(1):253-61

19. Day M. Covid-19: ibuprofen should not be used for managing symptoms, say doctors and scientists. BMJ. 2020 Mar 17;368:m1086.

20. Sodhi M, Etminan M. Safety of ibuprofen in patients with COVID-19: causa or confounded? Chest. 2020 Mar 31;31(3):516-24;

21. Chary MA, Barbuto AF, Izadmehr S, Hayes BD, Burns MM. COVID-19: therapeutics and their toxicities. J Med Toxicol. 2020 Apr 30;16(3):284-94

22. European Medicines Agency. EMA gives advice on the use of non-steroida anti-inflammatories for COVID-19. [Cited in 2020 March 25] Available from: https://www.ema.europa.eu/en/news/ema-gives-advice-use-non-steroidalanti-inflammatories-covid-19

23. Rhodes A, Evans LE, Alhazzani W, Levy MM, Antonelli M, Ferrer R, et al. Surviving sepsis campaign: international guidelines for management of sepsis and septic shock: 2016. Intensive Care Med. 2017;43(3):304-77.

24. Chen N, Zhou M, Dong X, Qu J, Gong F, Han Y. Epidemiological and clinical characteristics of 99 cases of 2019 novel coronavirus pneumonia in Wuhan, China: a descriptive study. Lancet. 2020;395(10223):507-13.

25. Tang N, Bai H, Chen X, Gong J, Li D, Sun Z. J Anticoagulant treatment is associated with decreased mortality in severe coronavirus disease 2019 patients with coagulopathy. Thromb Haemost. 2020 May; 18(5):1094-9.

26. Dweck MR, Bularga A, Hahn RT, Bing R, Lee KK, Chapman AR, et al. Globa evaluation of echocardiography in patients with COVID-19. Eur Heart J Cardiovasc Imaging. 2020 Jun 18;jeaa178. doi: 10.1093/ehjci/jeaa178. online ahead of print 